\title{
Financial Time Series Forecasting with Grouped Predictors using Hierarchical Clustering and Support Vector Regression
}

\author{
ZheGao $^{\mathrm{a}, \mathrm{b}, *}$ and JianjunYang ${ }^{\mathrm{c}}$ \\ a. Guanghua School of Management, Peking University, Beijing 100871, China \\ b. Peking University Founder Group, Beijing, 100871, China \\ c. Department of Intelligence Science, Center for Information Science, Room \\ 2314,Science Building 2, Peking University, Beijing 100871, China \\ truman.gz@gmail.com (ZheGao),yjjhyxy@gmail.com(Jianjun Yang)
}

\begin{abstract}
Financial time series prediction is regarded as one of the most challengingtasks due to the inherent noise and non-stationality of the data. This paperproposed a two-stage financial time series prediction approach hybridizingsupport vector regression (SVR) with hierarchical clustering (HC). By averaging the variables within the clusters obtained from hierarchical clustering, we define super predictors and use them as the input variables of the SVRforecasting model. Although averaging is a simple technique, it plays animportant role in reducing variance. To evaluate the performance of theproposed approach, the Shanghai-Shenzhen 300 index is used as illustrativeexample. The experimental results show that the proposed approach outperforms both the SVR model with principal component analysis and the SVRmodel with genetic algorithms in average prediction error and predictionaccuracy.
\end{abstract}

Keywords: Hierarchical clustering, Support vector regression, Financialtime series forecasting

\section{Introduction}

The financial market is one of the most complicated systems due to its undergoing constant evolution and non-linear dynamics. Financial timeseries forecasting is regarded as one of the most challenging tasks. Overthe last two decades, this subject has attracted numerous attention from investors, speculators and researchers [28, 29], and it has become an important issuein investment decision making.

Recently, growing attention has been paid to the use of large datasetsfor financial time series forecasting. Since both the availability of financialtime series data and the computational power to handle them have increasedtremendously, researchers are trying to enrich their forecasting models bytaking advantage of a broader information base. Conventional time seriesmodels are not suitable for incorporating a large number of predictors, so newmethods were developed. Since the existence of significant residual varianceof the actual stock return from the prediction of the regression equation, it ispossible that nonlinear models are suitable to explain this residual variance.Recently, the support vector machine (SVM), based on statistical learningtheory [13], has led to great potential and superior performance ina range of applications, including financial stock market prediction $[2,7,12,4,23-26]$. SVM is shown to be very resistant to the over-fitting problem and can

${ }^{*}$ Corresponding author. Tel.: (86)-10-68588329; (86)13520131418 
achieve a high generalization performance comparison to artificialneural networks. This is largely due to the unique theory of the structuralrisk minimization principle used to estimate a function by minimizing anupper bound of the generalization error. The solution of SVM, whose training process is equivalent to solving a constrained quadratic programmingproblem, is always unique and globally optimal. The weak points of neuralnetworks, such as over-fitting and getting stuck at local minima, have beensolved by SVM in theory. Support vector regression (SVR), the regressionmodel of SVM, deals with continuous data and has been receiving increasingattention to solve nonlinear regression problems $[1,7,19,20-22$, 27].

In the prediction of financial time series, another key problem is the inherent noise of the predictors, especially when the number of predictors is large.Learning SVR from the raw data with noise may torture the approximationfunction and result in the loss of generalization capability in the testing step. This is because not all of the pre-chosen predictors are informative or can provide high discrimination power. This is known as the curse of dimensionalityproblem [11]. In general, some relatedwork considers a feature selection step to examine the validity of their chosenvariables for effective stock prediction. Feature selection can lead to manypotential benefits, such as reducing data complexity and training time, etc.There are several feature selection methods, such as filter-based feature selection (FBFS) [1], genetic algorithms (GA) [14], principal component analysis (PCA) [6] and independent component analysis (ICA) [7]. However, in most applications, these methods have been applied to relativelysmall systems and that the relationship structure among the large time serieshave not been given enough attention in the literature.

In this paper, we present a way to improve the generalization capability of SVR through coupling with the hierarchical clustering method, andapplied it to financial time series forecasting. We first use hierarchical clustering on the predictors to obtain a dendrogram that reveals their nestedcorrelation structure. At each level of the hierarchy, we create a unique set of predictors and super predictors by computing the average expression of thecurrent clusters. The treatment here can overcome the collinearity problem among the predictors, which results in large variance of the estimates andinaccurate prediction [9]. Then we usedthe different sets of predictors and super predictors as inputs for SVR. Theempirical results show the efficiency of the proposed approach.

The rest of this paper is organized as follows: Section 2 gives a brief introduction about hierarchical clustering and support vector regression. Theproposed hybrid forecasting model is thoroughly described in Section 3. Theexperimental setup, including the chosen dataset and the process of constructing the prediction model and the evaluation methods is presented Section 4. Section 5 shows the experimental results and a conclusion is providedin Section 6.

\section{Methodology}

\subsection{Hierarchical Clustering}

Clustering analysis, one of the primary data analysis tasks in knowledgediscovery process, is used to identify valid, potentially useful, and understandable patterns in large amount of data. Hierarchical clustering is usedin our approach because it provides multiple levels at which the super predictors can be formed. Hierarchical clustering has several advantages overtraditional flat clustering, such as k-means. It is more robust with respectto the input parameters, less influenced by cluster shapes, less sensitive tolargely differing point densities of clusters, and more informative for datadisplay.

An agglomerative algorithm for hierarchical clustering is used in our paper. In order to decide which clusters should be combined for agglomerative, a measure of distance (or 
similarity) between sets of predictors is required.In most methods of hierarchical clustering, this is achieved by the using ofan appropriate metric (a measure of distance or similarity between pairs ofpredictors), and a linkage criterion which specifies pairwise distances of predictors in the sets. Correlation distance is used in our paper and is definedon couples of time series $(\mathrm{x}, \mathrm{y})$ as:

$$
d(\mathbf{x}, \mathbf{y})=\sqrt{2\left(1-c_{\mathbf{x y}}\right)}
$$

Where $\mathbf{x}=\left(x_{1}, x_{2}, \ldots, x_{T}\right), y=\left(y_{1}, y_{2}, \ldots, y_{T}\right)$ and $c_{\mathbf{x y}}$ is the correlation coefficient between $\mathbf{x}$ and $\mathbf{y}$.

There are several commonly used linkage criteria, such as single linkage,complete-linkage, average-linkage and minimum energy clustering. We use the average-linkage clustering and consider the distance between one clusterand another to be equal to the average distance. Given a set of $\mathrm{N}$ predictorsand an $\mathrm{N} \times \mathrm{N}$ similarity matrix, the basic process of hierarchical clusteringis implemented as follows [8]:

1. Start by assigning each predictor to a cluster. Set the distances (similarities) between the clusters equal to the distances (similarities) betweenthe predictors in the clusters.

2. Find the most similar pair of clusters and merge them into a singlecluster.

3. Compute distances (similarities) between the new cluster and each ofthe old clusters.

4. Repeat Steps 2 and 3 until all predictors are clustered into a singlecluster of size N.

\section{2. $\varepsilon$-Support Vector Regression}

For illustrating the concept of SVR, soft margin support vector regression ( $\varepsilon$-SVR) is explained and the nonlinear case is discussed in this section. Consider a set of training data $\mathrm{D}$ $=\left\{x_{i}, y_{i}\right\}_{i=1}^{n}$, in which $x_{i} \in R^{d}$ is the input vector, $y_{i}$ represents the corresponding scalar output, and $\mathrm{n}$ is total number of the data patterns. The objective of linear SVR is to build the regression function formulated as:

$$
f(x)=<\mathbf{w}, \mathbf{x}>+b ; w \in R^{d}, b \in R
$$

where $\langle\cdot, \cdot\rangle$ denotes the dot product in the input space. A loss function, (Eq. 3) known as a $\varepsilon$-insensitive function is usually used to evaluate the robustness of SVR.

$$
L_{\varepsilon}\left(f\left(x_{i}\right), y_{i}\right)=\left\{\begin{array}{cc}
0 & \text { if }\left|f\left(x_{i}\right)-y_{i}\right| \leq \varepsilon \\
\left|f\left(x_{i}\right), y_{i}\right|-\varepsilon & \text { otherwise }
\end{array}\right.
$$

The coefficients $\mathbf{w}$ and $b$ are estimated by minimizing

$$
R=\frac{1}{2}\|\mathbf{w}\|^{2}+C \frac{1}{n} \sum_{i=1}^{n} L_{\varepsilon}\left(f\left(x_{i}\right), y_{i}\right)
$$

In the risk function given by Eq. (4), the first term is the regularization term and the second term $C(1 / n) \sum_{i=1}^{n} L_{\varepsilon}\left(f\left(x_{i}\right), y_{i}\right)$ is the empirical risk, which is measured by the $\varepsilon$ -insensitive loss function givenby Eq. (3). $C$ is referred to as the regularized constant and is used to specify the trade-off between the regularization term and the empirical risk. Both $C$ and $\varepsilon$ are pre-defined parameters.

The deviation $L_{\varepsilon}\left(f\left(x_{i}\right), y_{i}\right)$ from the boundaries of the $\varepsilon$-insensitive zone can be measured by two positive slack variables, $\xi_{i}$ and $\xi_{i}^{*}, i=1,2, \ldots, n$. By introducing the slack variables, Eq. (4) is transformed into the following constrained form: 


$$
\begin{gathered}
\text { Minimize } R\left(\mathbf{w}, \xi, \xi^{*}\right)=\frac{1}{2}\|\mathbf{w}\|^{2}+C \sum_{i=1}^{n}\left(\xi_{i}+\xi_{i}^{*}\right) \\
\text { Subjected to } \quad y_{i}-f\left(x_{i}\right) \leq \varepsilon+\xi_{i}, \\
f\left(x_{i}\right)-y_{i} \leq \varepsilon+\xi_{i}^{*}, \\
\xi_{i} \geq 0, \xi_{i}^{*} \geq 0 .
\end{gathered}
$$

By applying the Lagrangian theory and the Karush Kuhn-Tucker (KKT) condition to this constrained optimization problem, it yields the corresponding dual form as follows:

$$
\begin{gathered}
\text { Maximize }-\varepsilon \sum_{i=1}^{n}\left(\alpha_{i}+\alpha_{i}^{*}\right)+\sum_{i=1}^{n} y_{i}\left(\alpha_{i}-\alpha_{i}^{*}\right)-\frac{1}{2} \sum_{i, j=1}^{n}\left(\alpha_{i}-\alpha_{i}^{*}\right)\left(\alpha_{j}-\alpha_{j}^{*}\right)<x_{i}, x_{j}>(6) \\
\text { Subjected to } \mathbf{e}^{T}\left(\left(\alpha_{i}-\alpha_{i}^{*}\right)\right)=0 \\
0 \leq \alpha_{i}, \alpha_{i}^{*} \leq C, i=1,2, \ldots, n
\end{gathered}
$$

where $\mathbf{e}=[1, \ldots, 1]^{T}$ is the vector of all ones, and $\alpha_{i}, \alpha_{i}^{*}$ are Lagrangeparameters. Eq. (6) represents a standard constrained quadratic programming problem, for which the common solution method is sequential minimaloptimization (SMO) algorithm [10]. By solving problem (6), the approximate function becomes:

$$
\hat{f}(x)=\sum_{i, j=1}^{n}\left(-\alpha_{i}+\alpha_{i}^{*}\right)<x_{i}, x>+b
$$

The next step is to extend the SVR algorithm to the nonlinear problem. This can be done by mapping the training patterns $x_{i}$ into a higher-dimensional feature space via a map function $\varnothing$. We could use an implicit mapping via kernels, which is computational cheaper. As noted above, the SVR algorithm only depends on dot products between patterns $x_{i}$, which can be replaced by scalar products $\left\langle\varnothing\left(x_{i}\right), \varnothing\left(x_{j}\right)>\right.$. The scalar product is calculated directly by computing a kernel function $K\left(x_{i}, x_{j}\right)$ to avoid performing a mapping $\varnothing(x)$. The radial basis function (RBF) is used in our paper, which is defined as $K\left(\mathbf{x}_{i}, \mathbf{x}_{j}\right)=\exp \left(-\gamma\left\|\mathbf{x}_{i}-\mathbf{x}_{j}\right\|^{2}\right)$, where $\gamma$ denotes the width of the RBF.

\section{The Proposed System Framework}

In this study, a two-stage hybrid method is implemented which combinesthe SVR prediction capability with the hierarchical clustering algorithm. Inthe first stage, HC clusters the original inputs to yield the nested correlationstructure. At each level of hierarchy, the correlated variables are automatically collected and the super predictors are created by averaging the variablesat each cluster. This gives $\mathrm{p}$ different sets of superpredictors that representeach level. In the second stage, an individual SVR model for each set ofthe superpredictors is constructed. In order to construct an accurate SVRmodel, the grid algorithm is adapted to find the proper parameters. Figure 1 shows detailed steps to train and test the two stage HC-SVR model.

Our method is advantageous when there are multiple variables with strong correlations. Specifically, if the sample correlations of the predictors are high enough, an averaged predictor lead to lower expected squared error of coefficient estimates. This fact has been proven by [9], who used the averaged features as inputs for Lasso regression. By averaging 
the original variables, the SVR model in our method yields a more accurate result than the individual predictors.

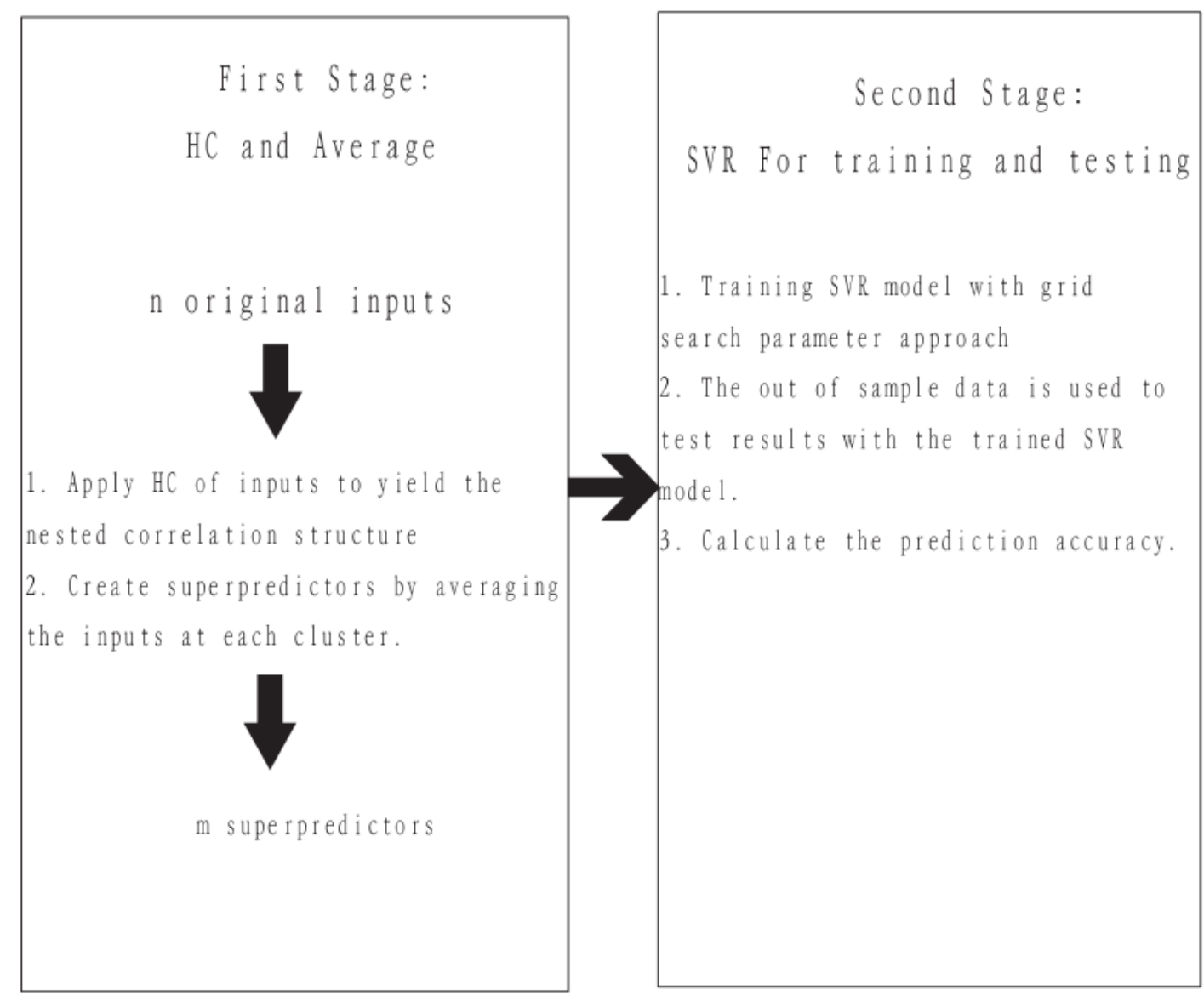

Figure 1. The Hybrid HC-SVR Architecture

\section{Experiment Design}

\subsection{Datasets and Variables}

For evaluating the performance of the proposed hybrid HC-SVR model, the Shanghai-Shenzhen 300 index (CSI300) of China is used in this study. The CSI300 selects 300 sweeping, liquid and most representative constituent stocks listed on the Shanghai and Shenzhen Stock Exchanges and is the first joint release of the A share market index.

Daily data from April 16, 2010 to January 31, 2012 with 433 observations are used in the evaluation, as shown in Figure 2. The sliding window is used to divide the sample data into different groups of training and testing data. The training set is composed of 10 months of samples and the testing set is based on the following month. For example, the training data of the first group is from the 20100416 to 20100131 and the testing data is from 20110201 to 20110228. Therefore, the model is trained and tested for 12 times. 


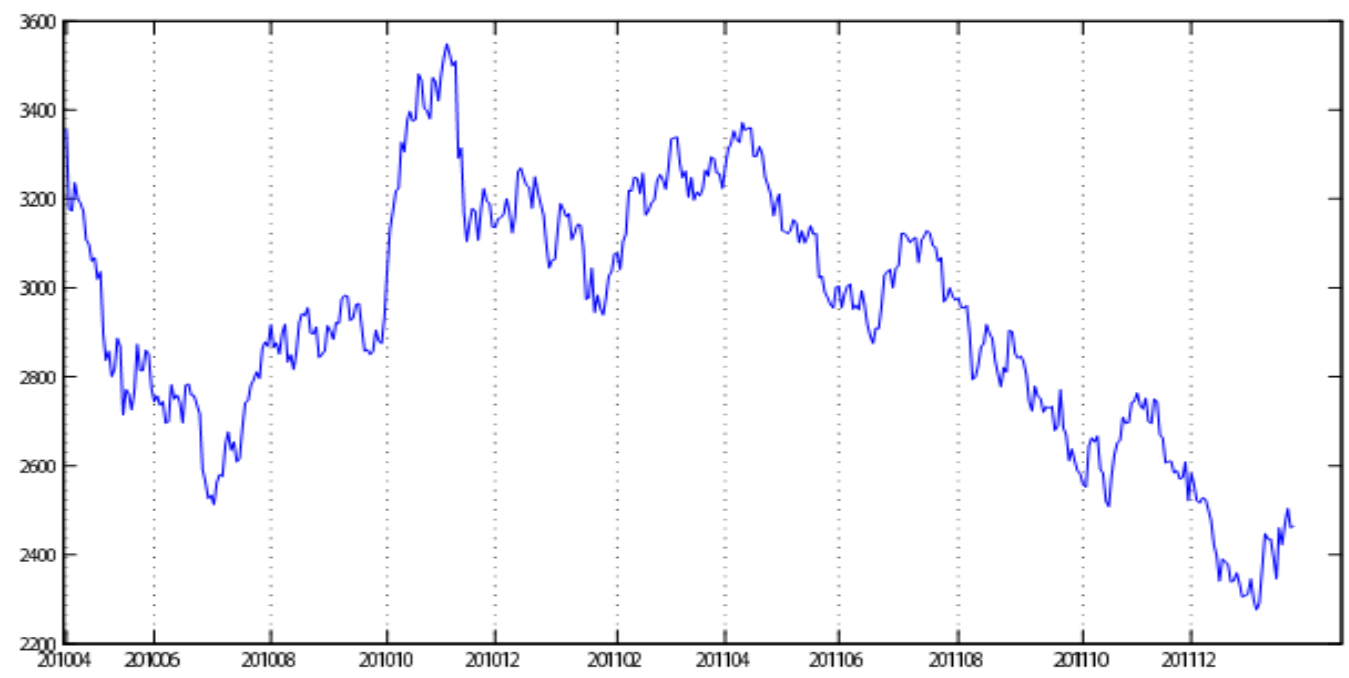

Figure 2. The CSI300 from January 5 to January 31, 2012

For forecasting the CSI300 closing prices, two groups of totally 26 indictors are used as forecasting variables. The first group are the 4 CSI300 indexfutures contracts traded on China Financial Futures Exchange (CFFE). Sincethe futures prices usually change before the market price [5], using the leading futures as inputs should contribute to the successin increasing the forecasting accuracy. The second group are 22 technical indicators determined by the review of domain experts and literatures, whichare the most widely used features in financial time series prediction. The descriptions and definitions of these input variables are presented in Appendix A.

\subsection{Evaluation Strategies}

The prediction performance is evaluated by four performance measures, namely, the root mean square error (RMSE), normalized mean square error (NMSE), mean absolute error (MAE), and directional symmetry (DS) (Table 1). RMSE, NMSE and MAE are measures of the deviation between actual and predicted values. The smaller values of RMSE, NMSE, MAE, the closer are the predicted time series values to that of the actual value. DS provides the correctness of the predicted direction of the CSI300 index in terms of percentage. A large value of DS suggests a better predictor.

Table 1. The Definitions of the Performance Measures

\begin{tabular}{|l|c|}
\hline Metrics & Definition \\
\hline RMSE & $R M S E=\sqrt{\frac{\sum_{i=1}^{n}\left(y_{i}-\hat{y}_{i}\right)^{2}}{n}}$ \\
\hline NMSE & $N M S E=\frac{\sum_{i=1}^{n}\left(y_{i}-\hat{y}_{i}\right)^{2}}{\sigma^{2} n}$, where $\sigma^{2}=\frac{\sum_{i=1}^{n}\left(y_{i}-\bar{y}\right)^{2}}{n-1}$ \\
\hline MAE & $M A E=\frac{\sum_{i=1}^{n}\left|y_{i}-\hat{y}_{i}\right|}{n}$ \\
\hline
\end{tabular}




$$
\text { DS } D S=\frac{100}{n} \sum_{i=1}^{n} d_{i}, \text { where } d_{i}=\left\{\begin{array}{cc}
1 & \left(y_{i}-y_{i-1}\right)\left(\hat{y}_{i}-\hat{y}_{i-1}\right) \geq 0 \\
0 & \text { otherwise }
\end{array}\right.
$$

\section{Experiment Results}

\subsection{Determining the Level of $\mathrm{HC}$}

Each level of hierarchical tree shows different performance. To determine the best level of HC, we use a threshold $\rho$, which is the minimum distance among the clusters in that level. The parameterpaffects the granularity of clustering and thenindirectly impacts on the goodness of the fit. To investigate the impacts of the number of clusters on the MAE performance, we construct the models for different value $\rho$. Figure 3 shows the MAE of HC-SVR at various $\rho$, in which $\mathrm{C}$ and $\varepsilon$ are respectively, fixed at 20 and 0.0005 . The figure shows that the MAE on the testing set decreases whenpincreases from 0 to 0.4 , but subsequently increases whilepincrease. This indicates that too large a value of $\rho(0.5-0.8)$ yields a larger bias to the solution of SVR. In the following experiments, we setpto 0.4.

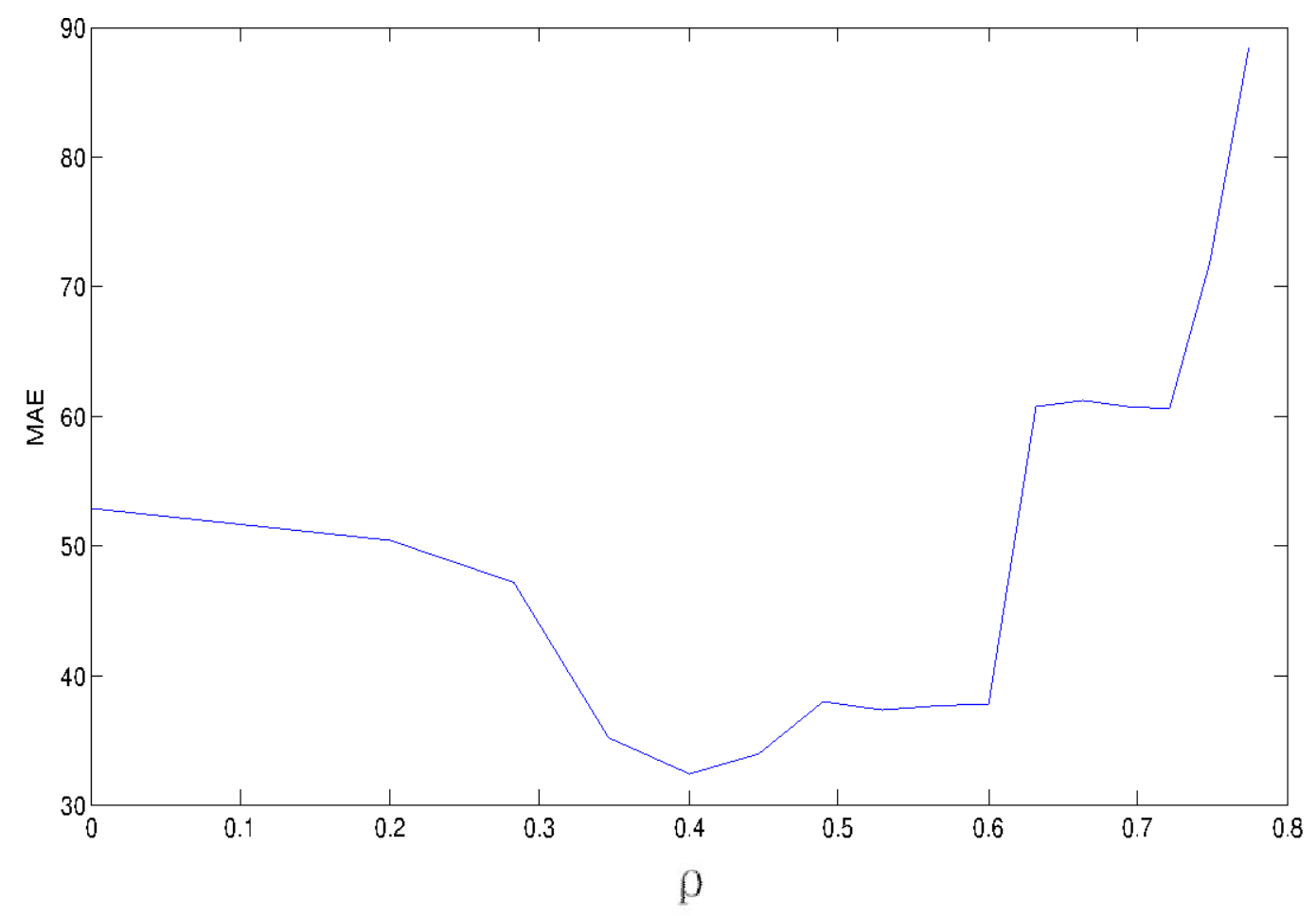

Figure 3. The Behavior of MAE in HC-SVR the MAE on the Testing Set Decreases when $\rho$ Increases from 0 to 0.4 , but Subsequently Increases while $\rho$ Increases

\subsection{Comparison}

This study compares the prediction accuracy of the HC-SVR method withPCA-SVR and GA-SVR, as PCA and GA are popular methods to select thefeatures. The differences among these three algorithms are firstly compared,which produces the inputs to the SVR model. Then we use the same RBFkernel, $€$-insensitive loss function and the grid search approach. 
To perform PCA-SVR, the question then is, how many factors do we needto extract various guidelines have been developed, and in this paper weuse the Kaiser criterion [3]. Specifically, we retain only factorswith eigenvalues greater than 1 , which means that unless a factor extractsat least as much as the equivalent of one original variable, we drop it. In ourexample, using this criterion we would retain 6 principal components.

To perform GA-SVR, we use the grid search algorithm to select the bestpossible results in terms of MAE. According to the prediction performance,the parameters used in this paper for comparisons are as follows: the population size is set to 25 , the crossover rate is set to 0.5 and mutation rate is set to 0.03 .

The performances of HC-SVR, PCA-SVR and GA-SVR on the test setare collated and the average results of 12 test sets are summarized in Table 2. It can be seen that HC-SVR has smaller RMSE, NMSE and MAEbut larger DS than PCA-SVR, GA-SVR. This indicates that HC-SVR out-performs PCA-SVR and GA-SVR in predicting CSI300, and it can forecastmore accurately and capture the turning points better than the others. Toevaluate the robustness of the proposed HC-SVR method, Fig. 4 shows theRMSE, NMSE, MAE and DS for the three models at each test set. As wecan see, for most of the test sets, the proposed HC-SVR method outperforms the other benchmarking tools. It therefore indicates that HC-SVRbased approach indeed provides better forecasting accuracy than the othertwo approaches.

Table 2. Averaged Results of HC-SVR, PCA-SVR and GA-SVR on the Test Set

\begin{tabular}{|c|c|c|c|c|}
\hline & RMSE & NMSE & MAE & DS \\
\hline HC-SVR & 41.079 & 0.018 & 32.686 & 55.319 \\
\hline PCA-SVR & 91.388 & 0.091 & 68.656 & 48.319 \\
\hline GA-SVR & 59.309 & 0.038 & 46.485 & 51.479 \\
\hline
\end{tabular}
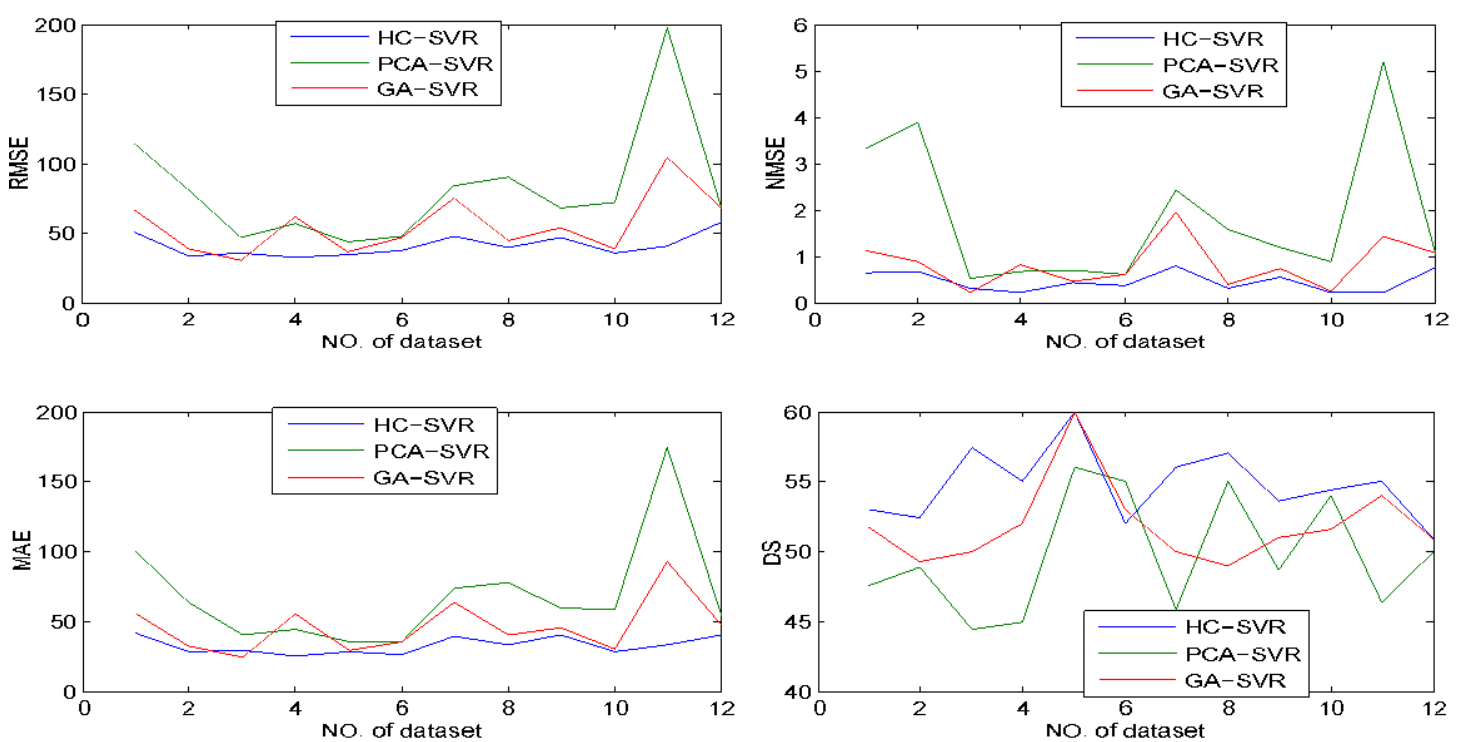

Figure 4. RMSE, NMSE, MAE, DS Comparisions: HC-SVR vs. PCA-SVR vs. GA-SVR ${ }^{*}$

*Note: The performances of HC-SVR, PCA-SVR and GA-SVR on the test set are collated and the average results 


\section{Conclusions}

In this study, we proposed a two-stage forecasting model by integrating HC and SVR for financial time series prediction. We first use hierarchical clustering to obtain the sets of correlated variables; then we average the variables within each cluster andinput the averages as superpredictors to SVR. When the variables are highly correlated, their average becomes a strong feature, yielding a fit with lower variance than the individual variables. To forecast CSI300, this study compares the proposed method with PA-SVR and GA-SVR using prediction error and prediction accuracy as the criteria. Experimental results showe that the proposed model can produce lower prediction error and higher prediction accuracy and outperformed the PA-SVR and GA-SVR.

Many problems in finance require the exploitation of large panels of time series. The idea proposed in this study can be extended to handle the large-dimensionality problem which could be solved with the development of parallel computing [15-18]. Future researches may integrating HC-SVR with other signal processing techniques,like nonnegative matrix factorization to improve the forecasting capabilities.

\section{Appendix A. Technical Indicator List}

1. OP,HP,LP,CP,VOL the intraday opening price, high price, low price, close price and volume, respectively

2. $\mathbf{M A}\left(\mathbf{C P , \mathbf { n } )}\right.$ n-day moving average, calculated as $M A(C P, n)_{t}=\sum_{i=t-n+1}^{t} C P_{i} / n$

3. $\operatorname{EMA}(\mathbf{C P}, \mathbf{n})$ n-day exponential moving average, calculated as $\operatorname{EMA}(C P, n)_{t}=\left(4 * \operatorname{EMA}(C P, n)_{t-1}+C P_{t}\right) / n$

4. $\quad \mathbf{R S I}(\mathbf{C P}, \mathbf{n})$ n-day relative strength index, calculated as $R S I(C P, n)_{t}=100 \times \operatorname{EMA}(U, n)_{t} /\left(\operatorname{EMA}(U, n)_{t}+\operatorname{EMA}(D, n)_{t}\right)$, where U, D are respectively upward and downward change for each trading period.

5. $\mathbf{C C I}(\mathbf{n})$ n-day commodity channel index, calculated as $C C I(n)=\left(p_{t}-M A(p, n)_{t}\right) /\left(0.015 * \sigma\left(p_{t}\right)\right)$, where the $p_{t}$ is the typical price, and $\sigma$ is the mean absolute deviation.

6. $\operatorname{PSY}(\mathbf{C P}, \mathbf{n})$ n-day psychological line, calculated as $P S Y(C P, n)_{t}=100 \times U D_{n} / n$, where $U D_{n}$ is the number of upward days during previous $n$ days.

7. $\quad \operatorname{VR}(\mathbf{n})$ n-day volume ratio, calculated as $V R(n)_{t}=100 \times(T H * 2+T Q) /(T L * 2+T Q)$, where $T H, T L, T Q$ are respectively the volume summation at upward days, downward days, and non-trend days.

8. WR(n) n-day Larry Williams' oscillator, calculated as $W R(n)_{t}=\left(H H V(H P, n)-C P_{t}\right) /(H H V(H P, n)-L L V(L P, n))$, where

of 12 test sets are summarized in Table 2. It can be seen that HC-SVR has smaller RMSE, NMSE and MAE but larger DS than PCA-SVR, GA-SVR. This indicates that HC-SVR outperforms PCA-SVR and GA-SVR in predicting CSI300, and it can forecast more accurately and capture the turning points better than the others. To evaluate the robustness of the proposed HC-SVR method, Fig. 4 shows the RMSE, NMSE, MAE and DS for the three models at each test set. As we can see, for most of the test sets, the proposed HC-SVR method outperforms the other benchmarking tools. It therefore indicates that HC-SVR based approach indeed provides better forecasting accuracy than the other two approaches. 
$H H V, L L V$ are respectively the highest high price and lowest low price in previous $n$ days period.

9. $\operatorname{BIAS}(\mathbf{n})$ n-day BIAS, calculated as $B I A S(n)_{t}=100 \times\left(C P_{t}-M A(C P, n)\right) / M A(C P, n)$

10. $\mathbf{A R}(\mathbf{n})$ n-day A ratio, calculated as

$$
A R(n)_{t}=\sum_{i=t-n+1}^{t}\left(H P_{i}-O_{i}\right) / \sum_{i=t-n+1}^{t}\left(O P_{i}-L P_{i}\right)
$$

11. $\mathbf{B R}(\mathbf{n})$ n-day $B$ ratio, calculated as

$$
B R(n)_{t}=\sum_{i=t-n+1}^{t}\left(H P_{i}-C P_{i-1}\right) / \sum_{i=t-n+1}^{t}\left(C P_{i-1}-L P_{i}\right)
$$

12. $\mathbf{K}(\mathbf{n}) \quad$ n-day stochastic index $\mathrm{K}$, calculated as

$$
\begin{aligned}
& K(n)_{t}=(n-1) / n \times K\left(n_{t-1}+1 / n \times R S V(n)_{t}\right), \text { where } \\
& R S V(n)_{t}=100 \times\left(C P_{t}-L L V(L P, n)\right) /(H H V(H P, n)-L L V(L P, n))
\end{aligned}
$$

13. D(n) n-day stochastic index $\mathrm{D}$, calculated as

$$
D(n)_{t}=(n-1) / n \times D(n)_{t-1}+1 / n \times K(n)_{t}
$$

14. DIF(short, long) the difference between a short-day and long-day exponential moving average, calculated as

$D I F(\text { short }, \text { long })_{t}=\operatorname{EMA}(C P, \text { short })_{t}-\operatorname{EMA}(C P, \text { long })_{t}$

15. DEA(n) n-day exponential moving average of $D I F$, calculated as $D E A(n)_{t}=\operatorname{EMA}(D I F, n)$

16. MACD moving average convergence/divergence, calculated as $M A C D_{t}=2 \times(D I F-D E A)$

17. ROC(n) n-day price change rate of change, calculated as $\operatorname{ROC}(n)_{t}=100 \times\left(C P_{t}-C P_{t-n}\right) / C P_{t-n}$

18. MTM(n) n-day momentum, calculated as $\operatorname{MTM}(n)_{t}=C P_{t}-C P_{t-n}$

\section{References}

[1] C. Huang and C. Tsai, "A hybrid sofm-SVR with a filter-based feature selection for stock market forecasting", Expert Systems with Applications, vol. 36, no. 2, (2009), pp. 1529-1539.

[2] W. Huang, Y. Nakamori and S. Wang, "Forecasting stock market movement direction with support vector machine", Computers \& Operations Research, vol. 32, no. 10, (2005), pp. 2513-2522.

[3] H. Kaiser, "The varimax criterion for analytic rotation in factor analysis", Psychometrika, vol. 23, no. 3, (1958), pp. 187-200.

[4] K. Kim, "Financial time series forecasting using support vector machines", Neuro computing, vol. 55, no. 1-2, (2003), pp. 307-319.

[5] T. Lee and N. Chen, "Investigating the information content of noncash-trading index futures using neural networks", Expert Systems with Applications, vol. 22, no. 3, (2002), pp. 225-234.

[6] X. Lin, Z. Yang and Y. Song, "Short-term stock price prediction based on echo state networks", Expert Systems with Applications, vol. 36, no. 3, (2009), pp. 7313-7317.

[7] C. Lu, T. Lee and C. Chiu, "Financial time series forecasting using independent component analysis and support vector regression", Decision Support Systems, vol. 47, no. 2, (2009), pp. 115-125.

[8] F. Murtagh, "A survey of recent advances in hierarchical clustering algorithms", The Computer Journal, vol. 26, no. 4, (1983), pp. 354-359.

[9] M. Park, T. Hastie and R. Tibshirani, "Averaged gene expressions for regression”, Biostatistics, vol. 8, no. 2, (2007), pp. 212-227.

[10] J. Platt, et al., "Sequential minimal optimization: A fast algorithm fortraining support vector machines", (1998). 
[11] M. Kaufmann, C. Saunders, A. Gammerman and V. Vovk, "Ridge regression learning algorithm in dual variables", In: Proceedings of the 15th International Conference on Machine Learning, vol. 19980, (1998), pp. 515-521, San Franscisco, CA, USA.

[12] F. Tay and L. Cao, "Application of support vector machines in financial time series forecasting", OMEGA-OXFORD-PERGAMON PRESS, vol. 29, (2001), pp. 309-317.

[13] V. Vapnik, "An overview of statistical learning theory”, Neural Networks, IEEE Transactions on, vol. 10, no. 5, pp. 988-999.

[14] L. Yu, S. Wang and K. Lai, "Mining stock market tendency using GA-basedsupport vector machines", Internet and Network Economics, (2005), pp. 336-345.

[15] Z. Jilin, L. Enyi, W. Jian, R. Yongjian, Y. Miao and W. Jue, "Implementing Sparse Matrix-Vector Multiplication with QCSR on GPU”, APPLIED MATHEMATICS \& INFORMATION SCIENCESVOL, vol. 7, no. 2, (2003), pp. 473-482.

[16] J.-L. Zhang, W. Qian, X.-H. Xu, J. Wan, Y.-Y. Yin and Y. J. Ren, "WLBS: A weight-based metadata server cluster load balancing strategy", International Journal of Advancements in Computing Technology, vol. 4, no. 1, (2004), pp. 77-85.

[17] J. L. Zhang, J. H. Zhang, H. Ding, J. Wan, Y. J. Ren and J. Wang, "Designing and Applying an Education IaaS System based on OpenStack", APPLIED MATHEMATICS \& INFORMATION SCIENCES, vol. 7, no. 2, (2007), pp. 155-160.

[18] J.-L. Zhang, Y. Chen, J. Wang, E.-Y. Liu, Y.-Y. Yin, Y.-J. Ren and C.-H. Li, "The implementation and optimization of irregular application task models based on the cell BE processor", International Journal of Digital Content Technology and its Applications, vol. 6, no. 2, (2012), pp. 269-278.

[19] C.-J. Lu and Y.-W. Wang, "Combining independent component analysis and growing hierarchical self-organizing maps with support vector regression in product demand forecasting", International Journal of Production Economics, vol. 128, Issue 2, (2010) December, pp. 603-613.

[20] B. Wang, H. Huang and X. Wang, "A novel text mining approach to financial time series forecasting”, Neuro computing, vol. 83, (2012) April 15, pp. 136-145.

[21] C.-S. Lin, S.-H. Chiu and T.-Y. Lin, "Empirical mode decomposition-based least squares support vector regression for foreign exchange rate forecasting", Economic Modeling, vol. 29, Issue 6, (2012) November, pp. 2583-2590.

[22] Y. Wang, B. Wang and X. Zhang, “A New Application of the Support Vector Regression on the Construction of Financial Conditions Index to CPI Prediction”, Procedia Computer Science, vol. 9, (2012), pp. 1263-1272.

[23] K.-J. Kim, "Financial time series forecasting using support vector machines", Neuro computing, ISSN 0925-2312, vol. 55, Issues 1-2, (2003) September, pp. 307-319.

[24] E. Francis, H. Tay and L. J. Cao, "Modified support vector machines in financial time series forecasting", Neuro computing, vol. 48, Issues 1-4, (2002) October, pp. 847-861.

[25] C.-J. Lu, T.-S. Lee and C.-C. Chiu, "Financial time series forecasting using independent component analysis and support vector regression", Decision Support Systems, vol. 47, Issue 2, (2009) May, pp. 115-125.

[26] S. Ismail, A. Shabri and R. Samsudin, "A hybrid model of self-organizing maps (SOM) and least square support vector machine (LSSVM) for time-series forecasting”, Expert Systems with Applications, vol. 38, Issue 8, (2011), August, pp. 10574-10578.

[27] Y. Ding, X. Song and Y. Zen, "Forecasting financial condition of Chinese listed companies based on support vector machine", Expert Systems with Applications, vol. 34, Issue 4, (2008), May, pp. 3081-3089.

[28] Z. Gao, J. Yang and S. Tan, "Forecasting Chinese GDP with mixed frequency data set: A generalized lasso granger method", Lecture Notes in Computer Science (including subseries Lecture Notes in Artificial Intelligence and Lecture Notes in Bioinformatics),LNCS, vol. 7929, no. 2, (2013), pp. 163-172.

[29] Z. Gao, Z. Wang, L. Wang and S. Tan, "Linear non-Gaussian causal discovery from a composite set of major US macroeconomic factors", Expert Systems with Applications, vol. 39, Issue 12, (2012) September 15, pp. 10867-10872. 
International Journal of Grid Distribution Computing Vol.7, No.5 (2014) 